PAPER

\title{
Vibration analysis of a musical drum head under nonuniform density and tension using a spectral method
}

\author{
Toshiya Samejima ${ }^{1, *}$ and Risa Fukuda ${ }^{2, \dagger}$ \\ ${ }^{1}$ Faculty of Design, Kyushu University, \\ 4-9-1, Shiobaru, Minami-ku, Fukuoka, 815-8540 Japan \\ ${ }^{2}$ Yamaha Corporation, \\ 10-1, Nakazawa-cho, Naka-ku, Hamamatsu, 430-8650 Japan
}

(Received 5 October 2015, Accepted for publication 20 June 2016)

\begin{abstract}
This paper develops a numerical method to analyze the membrane vibration of a membranophone with nonuniform heads, in which the density and tension vary smoothly. A spectral method is applied to numerically analyze the wave equation governing a membrane with spatially varying areal density and tension. An Indian drum tabla, representing nonuniform density, and a typical drum tom tom with several tension rods, representing nonuniform tension, are analyzed using the proposed numerical method to determine their eigenfrequencies and eigenmode shapes.
\end{abstract}

Keywords: Membranophone, Nonuniformity, Membrane vibration, Numerical analysis, Spectral method

PACS number: 43.40.Dx, 43.75.Hi [doi:10.1250/ast.37.295]

\section{INTRODUCTION}

The vibration of membranophone drum heads is usually analyzed on the assumption that the density and tension of the drum heads are uniform. However, real membranophones may have drum heads of nonuniform density and nonuniform tension. Moreover, some Indian membranophones, such as the tabla and mridangam, are intentionally fabricated using circular drum heads with a nonuniform density. By loading the central part of the drum head with material of a heavier density, its vibration can generate harmonic overtones [1]. As a result, such a membranophone has a strong sense of pitch and is distinctive from other general membranophones. In addition, timpani and snare drums are tuned by tightening their tension rods manually, thus their tensions are likely to be nonuniform. Furthermore, the Japanese musical drum, kotsuzumi, uses tension ropes (shirabeo) that are pulled by the player's left hand during performance [2], demonstrating that the tension of drum heads intentionally be made nonuniform.

A number of studies have previously been conducted in relation to eigenfrequencies. For example, Ramakrishna and Sondhi modeled a circular membrane under non-

*e-mail: samejima@design.kyushu-u.ac.jp

†e-mail: risa.fukuda@music.yamaha.com uniform density as a composite membrane of two distinct densities [3]. With this simplification and axisymmetric condition, they derived an eigenvalue equation for the eigenfrequencies of such a membrane. Ando and Yamaguchi investigated the structure of a Japanese musical drum, kotsuzumi, and determined that one of the heads has features of a loaded membrane with a mass in the center [4]. They used the same eigenvalue equation that Ramakrishna and Sondhi derived, and indicated that the eigenfrequencies of a kotsuzumi have harmonic properties.

Furthermore, Sathej and Adhikari used a spectral collocation method in eigenfrequency analysis to numerically analyze the vibration of a circular membrane under nonuniform density [5]. Their study demonstrated that the spectral collocation method can offer greater accuracy for this class of problem than the finite element method. Using this method, distribution of the areal density of the membrane that delivers maximally harmonic vibrations was obtained, for more harmonic tablas. Worland analyzed a circular drum head under nonuniform tension using perturbation theory and the finite element method [6]. However, for nonuniformity of the tension, the author only included angular dependence of the tension, and only considered symmetric tension perturbations for maintaining equilibrium. In addition, the tension perturbations were roughly discretized. We consider that one of reasons for assuming these limited conditions is that the study used 
commercial finite element analysis software, which limited the mathematical model. In fact, Worland noted in the paper that the goal was to make only qualitative (and not quantitative) comparisons of the mode shapes and frequencies in response to particular symmetric tension perturbations.

In the study presented in this paper, the drum head of a membranophone is modeled as a nonuniform membrane in which the density and tension vary smoothly, and a numerical method for calculating its vibration is developed. The main contribution of this work is that Sathej and Adhikari's numerical method for membrane vibration analysis is extended to include the nonuniformity of tension as well as that of density. To achieve this, we firstly derive the wave equation governing a membrane with spatially varying areal density and tension. In order to numerically analyze the wave equation, a spectral method, which offers greater accuracy for this class of problem than other numerical methods such as the finite element method, is adopted. The formulation of the developed numerical method is then confirmed through a comparison between numerical results and measured results presented in a previous paper. Through numerical calculations using the developed method, the nonuniformity effects of the density and tension of drum heads are then discussed quantitatively.

Through this paper, the time factor $\exp (j \omega t)$ is used, where $j=(-1)^{1 / 2}$ and $\omega$ denotes the angular frequency.

\section{PROPOSED NUMERICAL METHOD}

\subsection{Wave Equation for a Nonuniform Membrane}

Morse presented the most general equation of motion for the flexible string involving linear density, $\rho_{\mathrm{S}}(x)$, and tension of the string, $T_{\mathrm{S}}(x)[\mathrm{N}]$, both of which vary with $x$ [7], as

$$
\frac{\partial}{\partial x}\left\{T_{\mathrm{S}}(x) \frac{\partial \eta(x)}{\partial x}\right\}+\omega^{2} \rho_{\mathrm{S}}(x) \eta(x)=-f_{\mathrm{S}}(x),
$$

where $\eta(x)$ is the transverse displacement of the string and $f_{\mathrm{S}}(x)$ is the transverse applied force of density per unit length. To set up the wave equation for a nonuniform membrane, we simply add the derivative of the tension term with respect to $y$ to the above equation, and extend all the variables to functions of both $x$ and $y$ as

$$
\begin{aligned}
& \frac{\partial}{\partial x}\left\{T_{\mathrm{M}}(x, y) \frac{\partial \zeta(x, y)}{\partial x}\right\}+\frac{\partial}{\partial y}\left\{T_{\mathrm{M}}(x, y) \frac{\partial \zeta(x, y)}{\partial y}\right\} \\
& \quad+\omega^{2} \rho_{\mathrm{M}}(x, y) \zeta(x, y)=-f_{\mathrm{M}}(x, y),
\end{aligned}
$$

where $\rho_{\mathrm{M}}(x, y)$ is the spatially varying areal density, $T_{\mathrm{M}}(x, y)[\mathrm{N} / \mathrm{m}]$ is the spatially varying tension of the membrane, $\zeta(x, y)$ is the transverse displacement of the membrane, and $f_{\mathrm{M}}(x, y)$ is the transverse applied force of density per unit area. This procedure indicates that we confine our attention to membranes in which the tension is the same for every orientation, (i.e., membranes of an isotropic condition), but the value of the tension depends on the position in the membrane. This condition is assumed for the preliminary computational model in our work, although this assumption may not correspond exactly with that of a real condition. The spatial derivative term in the left-hand side of Eq. (2) is expanded as follows:

$$
\begin{gathered}
\frac{\partial}{\partial x}\left\{T_{\mathrm{M}}(x, y) \frac{\partial \zeta(x, y)}{\partial x}\right\}+\frac{\partial}{\partial y}\left\{T_{\mathrm{M}}(x, y) \frac{\partial \zeta(x, y)}{\partial y}\right\} \\
=T_{\mathrm{M}}(x, y)\left\{\frac{\partial^{2} \zeta(x, y)}{\partial x^{2}}+\frac{\partial^{2} \zeta(x, y)}{\partial y^{2}}\right\} \\
+\frac{\partial T_{\mathrm{M}}(x, y)}{\partial x} \frac{\partial \zeta(x, y)}{\partial x}+\frac{\partial T_{\mathrm{M}}(x, y)}{\partial y} \frac{\partial \zeta(x, y)}{\partial y}
\end{gathered}
$$

Through the chain rule, the above derivative is transformed into one in polar coordinates $(r, \theta)$, as follows:

$$
\begin{aligned}
T_{\mathrm{M}}(x, y) & \left\{\frac{\partial^{2} \zeta(x, y)}{\partial x^{2}}+\frac{\partial^{2} \zeta(x, y)}{\partial y^{2}}\right\} \\
& +\frac{\partial T_{\mathrm{M}}(x, y)}{\partial x} \frac{\partial \zeta(x, y)}{\partial x}+\frac{\partial T_{\mathrm{M}}(x, y)}{\partial y} \frac{\partial \zeta(x, y)}{\partial y} \\
= & T_{\mathrm{M}}(r, \theta)\left\{\frac{\partial^{2} \zeta(r, \theta)}{\partial r^{2}}+\frac{1}{r} \frac{\partial \zeta(r, \theta)}{\partial r}+\frac{1}{r^{2}} \frac{\partial^{2} \zeta(r, \theta)}{\partial \theta^{2}}\right\} \\
+ & \frac{\partial T_{\mathrm{M}}(r, \theta)}{\partial r} \frac{\partial \zeta(r, \theta)}{\partial r}+\frac{1}{r^{2}} \frac{\partial T_{\mathrm{M}}(r, \theta)}{\partial \theta} \frac{\partial \zeta(r, \theta)}{\partial \theta} .
\end{aligned}
$$

As a result, the wave equation governing a membrane with spatially varying areal density and tension is given with polar coordinates $(r, \theta)$, as

$$
\begin{gathered}
T_{\mathrm{M}}(r, \theta)\left\{\frac{\partial^{2} \zeta(r, \theta)}{\partial r^{2}}+\frac{1}{r} \frac{\partial \zeta(r, \theta)}{\partial r}+\frac{1}{r^{2}} \frac{\partial^{2} \zeta(r, \theta)}{\partial \theta^{2}}\right\} \\
+\frac{\partial T_{\mathrm{M}}(r, \theta)}{\partial r} \frac{\partial \zeta(r, \theta)}{\partial r}+\frac{1}{r^{2}} \frac{\partial T_{\mathrm{M}}(r, \theta)}{\partial \theta} \frac{\partial \zeta(r, \theta)}{\partial \theta} \\
+\omega^{2} \rho_{\mathrm{M}}(r, \theta) \zeta(r, \theta)=-f_{\mathrm{M}}(r, \theta) .
\end{gathered}
$$

The first term on the left-hand side of Eq. (5) expresses the spatial derivative term of the conventional wave equation for a membrane, and the second and third terms express the corrective terms that must be included when the tension of a membrane varies smoothly.

\subsection{Application of a Spectral Method}

As referred to in the Introduction, it is acknowledged that using spectral collocation methods offers the greatest accuracy for the least computational expense in this class of problem [5]. Thus, we adopt a Fourier-Chebyshev spectral collocation technique [8] to numerically analyze the wave equation for a nonuniform membrane. Using the Chebyshev points $\tilde{r}_{i}=\cos \left(i \pi / N_{r}\right), i=0,1, \ldots, N_{r}$, for computational space $\tilde{r} \in[+1,-1]$, the Chebyshev differentiation matrix 


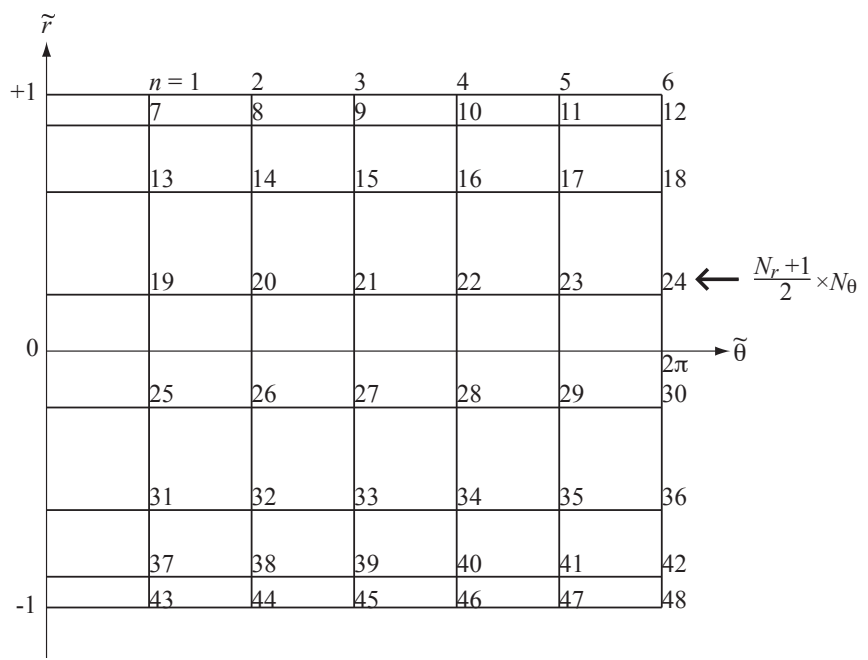

Fig. 1 Tensor product grid in $(\tilde{r}, \tilde{\theta})$ space and its global node number $n$, when $N_{r}=7$ and $N_{\theta}=6$.

$$
\boldsymbol{D}_{r}=\left[\begin{array}{ll}
\boldsymbol{E}_{1} & \boldsymbol{E}_{2} \\
\boldsymbol{E}_{3} & \boldsymbol{E}_{4}
\end{array}\right]
$$

is constructed. $\boldsymbol{E}_{1}, \quad \boldsymbol{E}_{2}, \boldsymbol{E}_{3}, \quad \boldsymbol{E}_{4}$ are block matrices corresponding to $\tilde{r}_{i}>0$ or $\tilde{r}_{i}<0$. $\boldsymbol{D}_{r}$ represents the first derivative with respect to $\tilde{r}$. To compute the second derivative with respect to $\tilde{r}$, we simply square $\boldsymbol{D}_{r}$.

$$
\boldsymbol{D}_{r}^{2}=\left[\begin{array}{ll}
\boldsymbol{D}_{1} & \boldsymbol{D}_{2} \\
\boldsymbol{D}_{3} & \boldsymbol{D}_{4}
\end{array}\right]
$$

For the computational space $\tilde{\theta} \in[0,2 \pi]$, periodic grid points $\tilde{\theta}_{j}=2 j \pi / N_{\theta}, j=1,2, \ldots, N_{\theta}$ are used, and the spectral differentiation matrix, $\boldsymbol{D}_{\theta}$, is constructed. The detailed mathematical procedures used to construct $\boldsymbol{D}_{r}$ and $\boldsymbol{D}_{\theta}$ are available in Ref. [8].

In order to construct the differentiation matrix in the polar $(\tilde{r}, \tilde{\theta})$ two-dimensional computational space, we set up a grid known as a tensor product grid, that is based on Chebyshev points in the $\tilde{r}$-direction and periodic grid points in the $\tilde{\theta}$-direction [8]. Figure 1 shows the tensor product grid and global numbering of its nodes in the case where $N_{r}=7$ and $N_{\theta}=6$. Note that, the first half of nodal values, i.e., those corresponding to $\tilde{r}_{i}>0$, can replace the last half of nodal values due to a symmetry condition in $(\tilde{r}, \tilde{\theta})$ space:

$$
\zeta(\tilde{r}, \tilde{\theta})=\zeta(-\tilde{r},(\tilde{\theta}+\pi)) .
$$

Thus, we in practice discard the last half of nodes, i.e., those corresponding to $\tilde{r}_{i}<0$. In addition to $\boldsymbol{D}_{r}$ and $\boldsymbol{D}_{\theta}$, the matrices below are also prepared.

$$
\begin{aligned}
& \boldsymbol{R}=\operatorname{diag}\left[\frac{1}{\tilde{r}_{i}}\right], \quad i=0,1, \ldots, \frac{N_{r}-1}{2}, \\
& \boldsymbol{T}=\operatorname{diag}\left[\left.T(\tilde{r}, \tilde{\theta})\right|_{n}\right], \quad n=1,2, \ldots, \frac{N_{r}+1}{2} \times N_{\theta},
\end{aligned}
$$

$$
\boldsymbol{T}_{r}=\operatorname{diag}\left[\left.\frac{\partial T(\tilde{r}, \tilde{\theta})}{\partial \tilde{r}}\right|_{n}\right], \quad \boldsymbol{T}_{\theta}=\operatorname{diag}\left[\left.\frac{\partial T(\tilde{r}, \tilde{\theta})}{\partial \tilde{\theta}}\right|_{n}\right],
$$

where $\left.T(\tilde{r}, \tilde{\theta})\right|_{n}$ indicates the value of $T$ at the $n$-th node. Using these matrices, with $\tilde{r}=r / a, \tilde{\theta}=\theta$ where $(r, \theta)$ is physical space and $a$ is the radius of a circular membrane, the differential operator in the left-hand side of Eq. (5) is replaced by the Fourier-Chebyshev differentiation matrix

$$
\begin{aligned}
\boldsymbol{L}^{\prime}= & \frac{1}{a^{2}} \boldsymbol{T} \boldsymbol{L}+\frac{1}{a^{2}} \boldsymbol{T}_{r}\left[\boldsymbol{E}_{1} \otimes\left[\begin{array}{ll}
\boldsymbol{I} & \mathbf{0} \\
\mathbf{0} & \boldsymbol{I}
\end{array}\right]+\boldsymbol{E}_{2}^{\prime} \otimes\left[\begin{array}{ll}
\mathbf{0} & \boldsymbol{I} \\
\boldsymbol{I} & \mathbf{0}
\end{array}\right]\right] \\
& +\frac{1}{a^{2}} \boldsymbol{T}_{\theta}\left[\boldsymbol{R}^{2} \otimes \boldsymbol{D}_{\theta}\right]
\end{aligned}
$$

with

$$
\begin{aligned}
\boldsymbol{L}= & {\left[\boldsymbol{D}_{1}+\boldsymbol{R} \boldsymbol{E}_{1}\right] \otimes\left[\begin{array}{ll}
\boldsymbol{I} & \mathbf{0} \\
\mathbf{0} & \boldsymbol{I}
\end{array}\right] } \\
& +\left[\boldsymbol{D}_{2}^{\prime}+\boldsymbol{R} \boldsymbol{E}_{2}^{\prime}\right] \otimes\left[\begin{array}{ll}
\mathbf{0} & \boldsymbol{I} \\
\boldsymbol{I} & \mathbf{0}
\end{array}\right] \\
& +\boldsymbol{R}^{2} \otimes \boldsymbol{D}_{\theta}^{2},
\end{aligned}
$$

where $\boldsymbol{I}$ is the $N_{\theta} / 2 \times N_{\theta} / 2$ identity matrix, $\otimes$ indicates the Kronecker product, and $\boldsymbol{D}_{2}^{\prime}$ and $\boldsymbol{E}_{2}^{\prime}$ respectively express these matrices with columns flipped in the left-right direction, i.e., about a vertical axis. The loading function, $\rho_{\mathrm{M}}(\tilde{r}, \tilde{\theta})$, is also replaced by a mass matrix

$$
\boldsymbol{B}=\operatorname{diag}\left[\left.\rho_{\mathrm{M}}(\tilde{r}, \tilde{\theta})\right|_{n}\right], \quad n=1,2, \ldots, \frac{N_{r}+1}{2} \times N_{\theta} .
$$

Using matrices (10) and (11), the wave equation (5) can then be formulated as a matrix equation,

$$
\boldsymbol{L}^{\prime} \boldsymbol{\zeta}+\omega^{2} \boldsymbol{B} \zeta=-\boldsymbol{f}
$$

where $\zeta$ is a vector with components of $\zeta(r, \theta)$ at each node and $\boldsymbol{f}$ is a vector with components of $f(r, \theta)$ at each node.

\section{VIBRATION ANALYSIS OF NONUNIFORM DENSITY DRUM HEADS}

Figure 2 shows a popular Indian drum, tabla. The drum head is made with goatskin and is loaded at the center. The central loading patch, the sihai, is made of a paste of soot, iron filings, and flour, and applied layer by layer to the goatskin membrane. Remarkably, the low eigenmodes of such a nonuniform membrane are harmonic in contrast with that of a circular membrane with a uniform density. The drum head is modeled as a nonuniform membrane with a density that varies smoothly, and its eigenmodes and eigenfrequencies are calculated using the proposed numerical method. The numerical method used in this section is the same as that applied in previous work [5]. However, in the previous work, the parametric study of the number of grid points was insufficient, and therefore we explore the relation between the number of grid points and the accuracy of the proposed numerical method in more detail, 


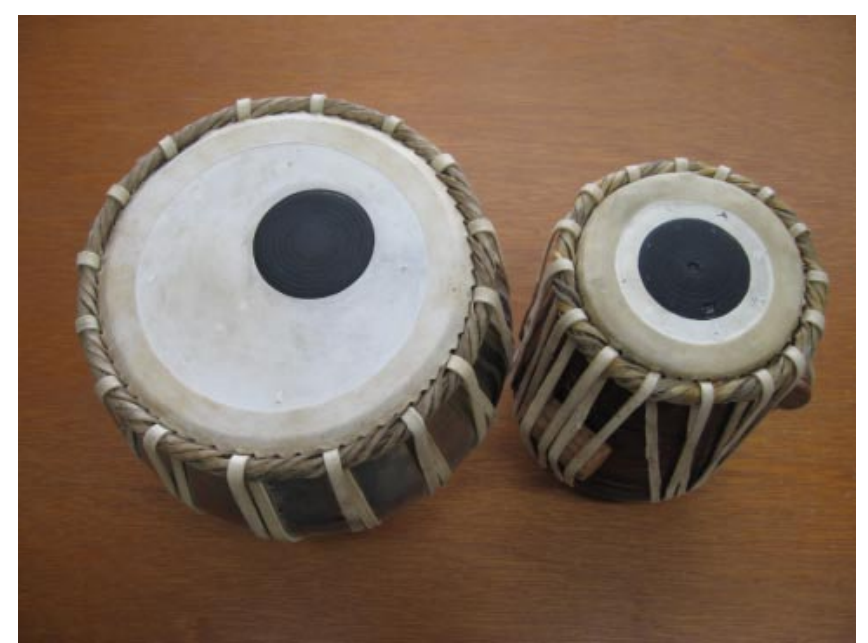

Fig. 2 Popular Indian drum tabla.

particularly by making an aspect of comparison with experimental data.

\subsection{Conditions of the Analysis}

The problem was solved using the following characteristics. The membrane used for analysis was fastened along its boundary circle, i.e., the transverse displacement $\zeta(\tilde{r}=$ 1) was forced to zero. The radius, $a$, of the membrane was taken as $0.1778 \mathrm{~m}$, and the uniform tension, $T$, of the membrane was set at $2,700 \mathrm{~N} / \mathrm{m}$. A function was chosen for the areal density $\rho_{\mathrm{M}}(\tilde{r}, \tilde{\theta})$ [5] that enabled a smooth change between the value at the circumference and that at the center of the loaded region, as

$$
\rho_{\mathrm{M}}(\tilde{r}, \tilde{\theta})=\rho_{2}\left[1+\frac{\left(\sigma^{2}-1\right)}{2}\left\{1-\tanh \left(\frac{R(\tilde{r}, \tilde{\theta})-k}{\xi}\right)\right\}\right]
$$

with

$$
R(\tilde{r}, \tilde{\theta})=\sqrt{(\tilde{r} \cos \tilde{\theta}-\varepsilon)^{2}+(\tilde{r} \sin \tilde{\theta})^{2}} .
$$

This function changes smoothly from a value of $\rho_{2}$ at $\tilde{r}=1$ to a value of $\rho_{1}=\rho_{2} \sigma^{2}$ at the center of the membrane. The change occurs over a region of the width $\xi$ along a circle with an equation $\tilde{r}=R(\tilde{r}, \tilde{\theta})$. For $\varepsilon=0 \rho_{\mathrm{M}}(\tilde{r}, \tilde{\theta})$ is radially symmetric. In addition, $k$ is the ratio of the radii of the loaded region. Figure 3 illustrates the variation of the function $\rho_{\mathrm{M}}(\tilde{r}, \tilde{\theta})$ for $\rho_{2}=0.3 \mathrm{~kg} / \mathrm{m}^{2}, \quad \sigma=3.125, \xi=$ $0.091, \varepsilon=0$, and $k=0.4$, which were used in the previous work [5] for comparison with experimental values. Using these parameters, the generalized eigenvalue problem, which is derived from Eq. (12) with $f=0$, was solved for the eigenvectors and eigenvalues.

First, the convergence characteristics of eigenfrequencies calculated by the proposed numerical method were investigated. The eigenfrequencies were calculated for several values of the number $N_{r}$ and $N_{\theta}$ of grid points, and

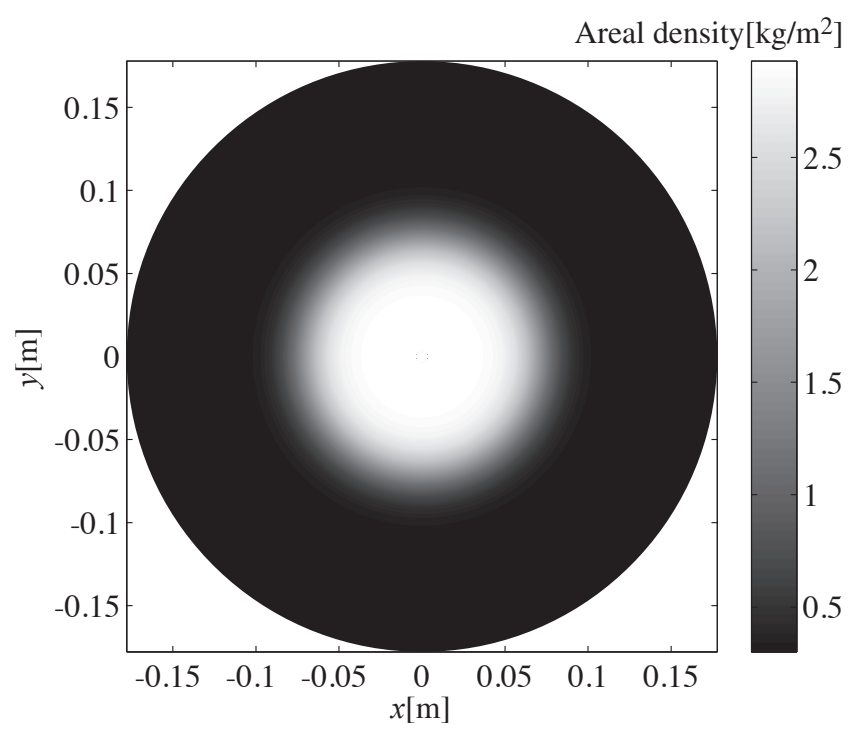

Fig. 3 Variation in areal density of the membrane.

then compared with those calculated using a very large number of grid points, $N_{r}=91$ and $N_{\theta}=90$. We have surely confirmed that the number of grid points generates sufficiently convergent numerical values of eigenfrequencies. The convergence characteristics were then evaluated by the averaged relative errors $E_{\mathrm{c}}$ of the numerical eigenfrequencies, given by

$$
E_{\mathrm{c}}=\frac{1}{N_{\max }} \sum_{i=1}^{N_{\max }} \frac{\left|\omega_{i}^{\mathrm{cn}}-\omega_{i}^{\mathrm{sp}}\right|}{\omega_{i}^{\mathrm{cn}}},
$$

where $\omega_{i}^{\mathrm{cn}}$ denotes the $i$-th eigenfrequency calculated for $N_{r}=91$ and $N_{\theta}=90$ (i.e., convergent numerical eigenfrequency); $\omega_{i}^{\mathrm{sp}}$ is $i$-th eigenfrequency calculated by the proposed numerical method; $i$ is the rank of the eigenmode when sorted in ascending order of eigenfrequencies; and $N_{\text {max }}$ is the number of eigenfrequencies used for calculating $E_{\mathrm{c}}$. The first nine experimental eigenfrequencies are given in Ramakrishna and Sondhi's paper [3], and therefore $N_{\text {max }}=9$ was adopted also here.

Next, we benchmarked our code implementing the proposed numerical method with the eigenfrequencies determined from Ramakrishna and Sondhi's experiment [3]. Their experiment used a number of commercially made tablas, and the bottom of the wooden shell of these tablas was opened to reduce any reaction between the air in the shell and the drum head. They measured the averaged eigenfrequencies of these tablas for a number of different tensions. Therefore, we consider that their experimental eigenfrequencies are very reliable for use as reference values. Using the proposed numerical method, eigenfrequencies were calculated for several values of the number $N_{r}$ and $N_{\theta}$ of grid points, and the averaged relative errors of the numerical eigenfrequencies were then calculated. The averaged relative error, $E_{\mathrm{e}}$, in this case is given by 
Table 1 Normalized eigenfrequencies of the membranes with uniform and nonuniform density. The frequencies are normalized by the $(1,1)$ mode.

\begin{tabular}{lllll}
\hline Modal Index & $\begin{array}{l}\text { Uniform } \\
\text { (Theoreti- } \\
\text { cal } \\
\text { values) }\end{array}$ & $\begin{array}{l}\text { Uniform } \\
\text { (Spectral } \\
\text { method) }\end{array}$ & $\begin{array}{l}\text { Non- } \\
\text { uniform } \\
\text { (Spectral } \\
\text { method) }\end{array}$ & $\begin{array}{l}\text { Non- } \\
\text { uniform } \\
\text { (Experi- } \\
\text { mental } \\
\text { values) }\end{array}$ \\
\hline$(0,1)$ & 1.2552 & 1.2552 & 1.0344 & 1.03 \\
$(1,1)_{\text {even,odd }}$ & 2 & 2 & 2 & 2 \\
$(2,1)_{\text {even,odd }}$ & 2.6806 & 2.6806 & 3.0542 & 3 \\
$(0,2)$ & 2.8813 & 2.8813 & 3.0395 & 3 \\
$(3,1)_{\text {even,odd }}$ & 3.3302 & 3.3302 & 4.0109 & 4 \\
$(1,2)_{\text {even,odd }}$ & 3.6619 & 3.6619 & 4.1487 & 4 \\
$(4,1)_{\text {even,odd }}$ & 3.9608 & 3.9608 & 5.0034 & 5.03 \\
$(2,2)_{\text {even,odd }}$ & 4.3935 & 4.3935 & 5.2528 & 5.08 \\
$(0,3)$ & 4.5169 & 4.5169 & 4.7655 & 5.04 \\
\hline
\end{tabular}

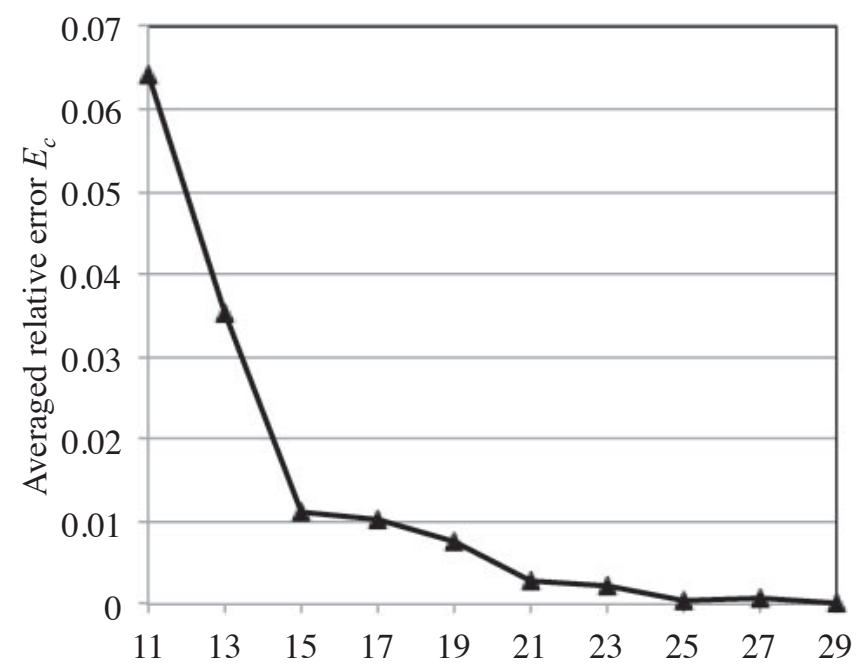

The number $N_{r}$ of grid points in the $r$-direction

Fig. 4 Averaged relative error of the calculated eigenfrequencies to the convergent eigenfrequencies as a function of the number of grid points in the $r$-direction.

$$
E_{\mathrm{e}}=\frac{1}{N_{\max }} \sum_{i=1}^{N_{\max }} \frac{\left|\omega_{i}^{\mathrm{ex}}-\omega_{i}^{\mathrm{sp}}\right|}{\omega_{i}^{\mathrm{ex}}},
$$

where $\omega_{i}^{\text {ex }}$ denotes the $i$-th eigenfrequency identified experimentally by Ramakrishna and Sondhi. The experimental eigenfrequencies are presented in the extreme right column of Table 1; it is of note that all frequencies are normalized by the frequency of the $(1,1)$ mode taken as being equal to 2 .

\subsection{Results}

Figure 4 shows the variation in $E_{\mathrm{c}}$ with an increase in $N_{r}$, at a fixed value of $N_{\theta}=30$. Although we calculated $E_{\mathrm{c}}$ for $N_{\theta}=40,50$, and 60 , the values of $E_{\mathrm{c}}$ were almost the same as that for $N_{\theta}=30$. While one can see a considerable decrease of $E_{\mathrm{c}}$ with an increase in $N_{r}$ from 11 to 15 , the rate

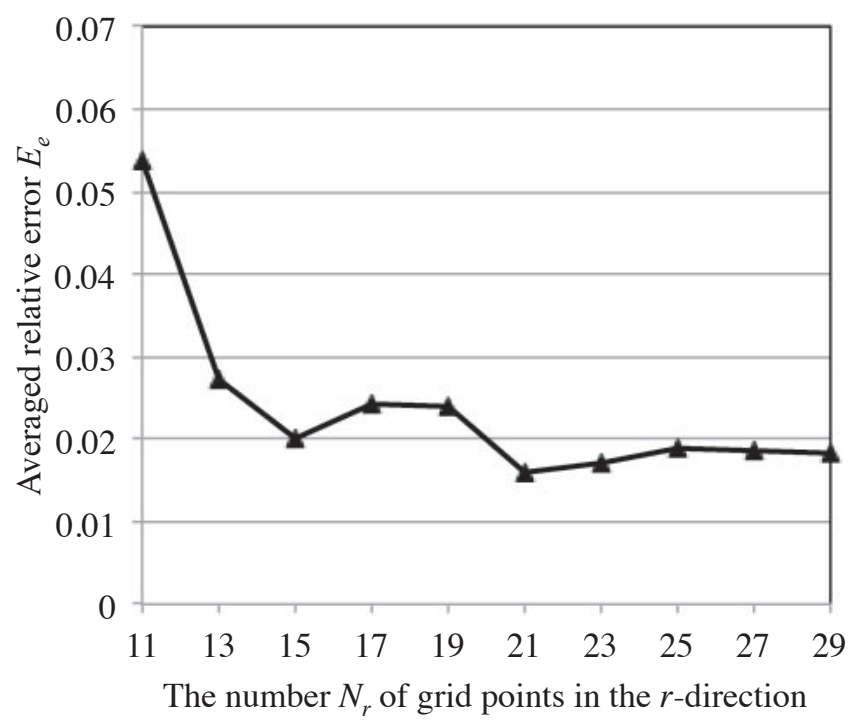

Fig. 5 Averaged relative error of the calculated eigenfrequencies to the experimental eigenfrequencies as a function of the number of grid points in the $r$-direction.

of the decrease becomes small when $N_{r}$ is increased from 15 to 19 . In addition, when $N_{r}$ is greater than 23 , the value of $E_{\mathrm{c}}$ is almost equal to zero; reliable calculations are carried out from the standpoint of numerical convergence.

Figure 5 shows the variation of $E_{\mathrm{e}}$ with an increase in $N_{r}$, at a fixed value of $N_{\theta}=30$. It can be seen that, $E_{\mathrm{e}}$ shows a tendency to fluctuate until $N_{r}$ reaches the value of 23. When $N_{r}$ is greater than 23, the value of $E_{\mathrm{e}}$ levels off at about 0.018 .

From these parametric studies of the number of grid points, we consider that the grid points generated by $N_{r}=$ 25 and $N_{\theta}=30$ can yield an acceptable accuracy of the numerical eigenfrequencies while the number of the grid points is rather small. It is also considered that the limit value of the relative error between the numerical and experimental eigenfrequencies is about 0.018 .

We also suppose that instability in the accuracy observed, especially in the range from $N_{r}=15$ to 21 , is attributed to the position of grid points in the $r$-direction. Figure 6 shows the variation in nonuniform areal density of the membrane calculated using Eq. (13), with the position of the Chebyshev points in the $r$-direction for $N_{r}=15,17$, and 21. To conduct reliable calculations, it is probably necessary to arrange each grid point at the position of each reasonable anchor point that can exactly express the variation in nonuniform areal density.

In Table 1 we compare the eigenfrequencies of a membrane with a uniform density and those of the membrane with a nonuniform density, calculated using the proposed numerical method. The uniform areal density, $\rho_{\mathrm{M}}$, of the membrane was set to $0.3 \mathrm{~kg} / \mathrm{m}^{2}$. For mesh generation of the spectral method, $N_{r}=25$ and $N_{\theta}=30$, 

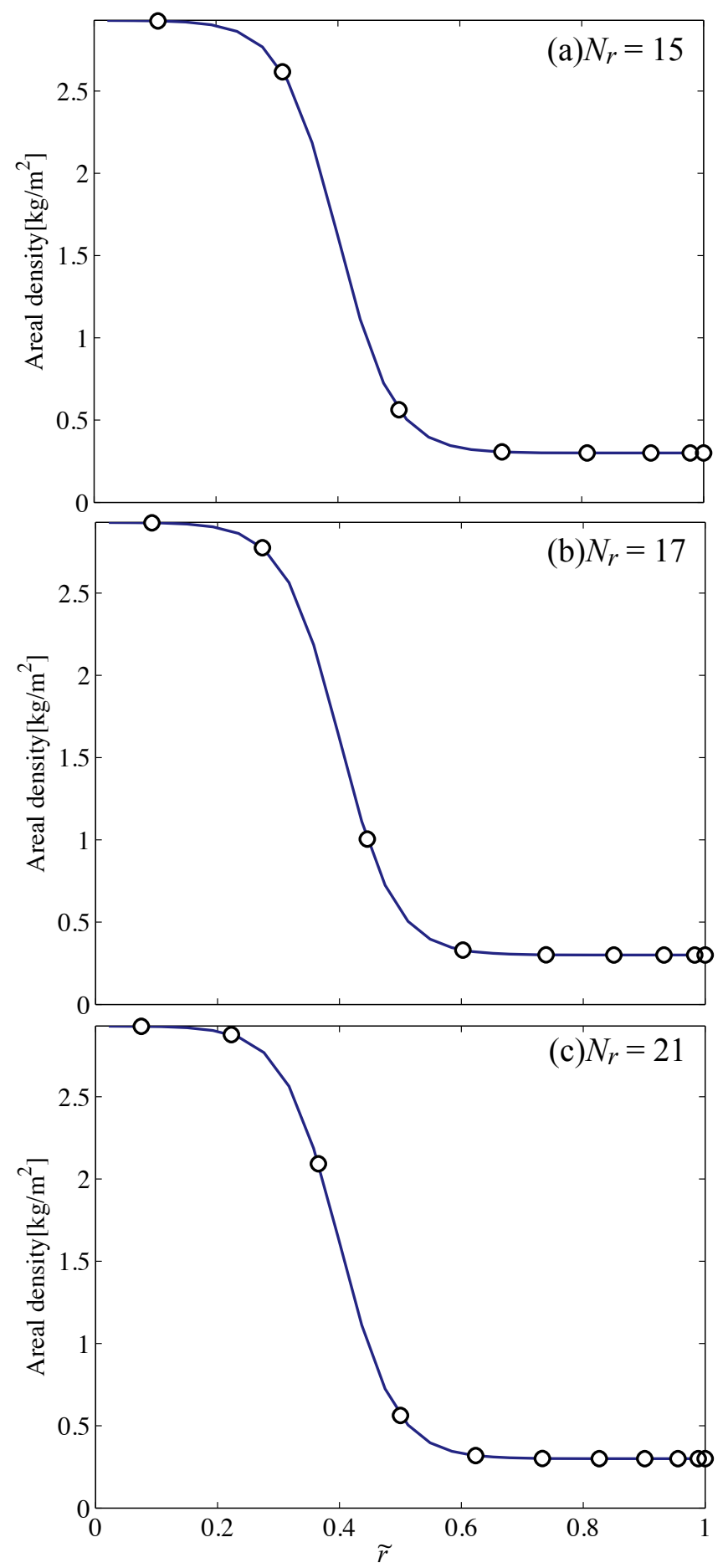

Fig. 6 Relation between the areal density of membrane (solid line) and the position of Chebyshev points in the $r$-direction (open circle).

which resulted in the reliable calculations, were used. We also calculated theoretical values of the eigenfrequencies of the uniform membrane, and these values are shown in the extreme left column of the table. In the uniform membrane, the eigenfrequencies calculated by the proposed numerical method have very good agreement with the theoretical values. It is clear that the eigenfrequencies of the nonuniform membrane are very close being in integer ratios,

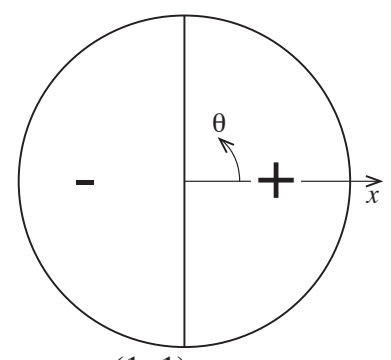

$(1,1)$ even

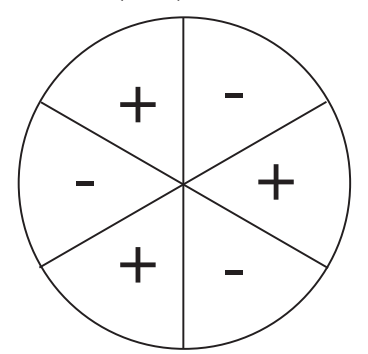

$(3,1)$ even

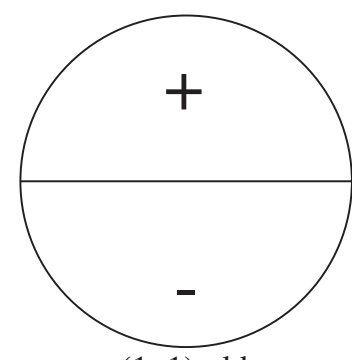

$(1,1)$ odd

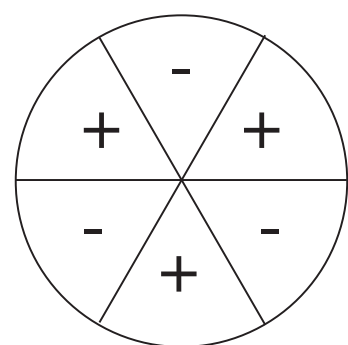

$(3,1)$ odd
Fig. 7 Modes of an ideal circular membrane with the modal indices $(m, n)$. "even(odd)" means that the mode shape is an even(odd) function in the $\theta$-axis.

while those of the uniform membrane are not harmonic. This phenomenon is attributed to the interesting musical property of membranophones of this type. It is thus possible that a more attractive tabla could be designed through use of the proposed numerical method.

\section{VIBRATION ANALYSIS OF NONUNIFORM TENSION DRUM HEADS}

Figure 7 shows a few of the mode shapes of an ideal circular membrane with $(m, n)$ indices. Each of these modes has a partner with the same frequency and shape, rotated by an angle $\pi / 2 m$ (even mode and odd mode); any mode involving at least one nodal diameter $(m>0)$ is doubly degenerate. This degeneracy may be lifted by a variety of perturbations, including nonuniform tension. Typical drums (as shown in Fig. 8) have drum heads involving nonuniform tension, because their heads are held taut by several tension rods that are placed at different and equidistant positions in the drum shell and are tightened separately. Striking such a nonuniform drum head at different locations will selectively excite its even mode or odd mode. Striking antinodes of an even mode causes the lower (higher) frequency to be heard, while striking antinodes of an odd mode causes the higher (lower) frequency to be heard. Striking at regions between a nodal line of an even mode and that of an odd mode will excite both modes, thereby leading to the production of audible beats. These audible frequency-splitting phenomena are of practical concern to drummers for "clearing the head." In this section, these phenomena are analyzed using the proposed numerical method. 


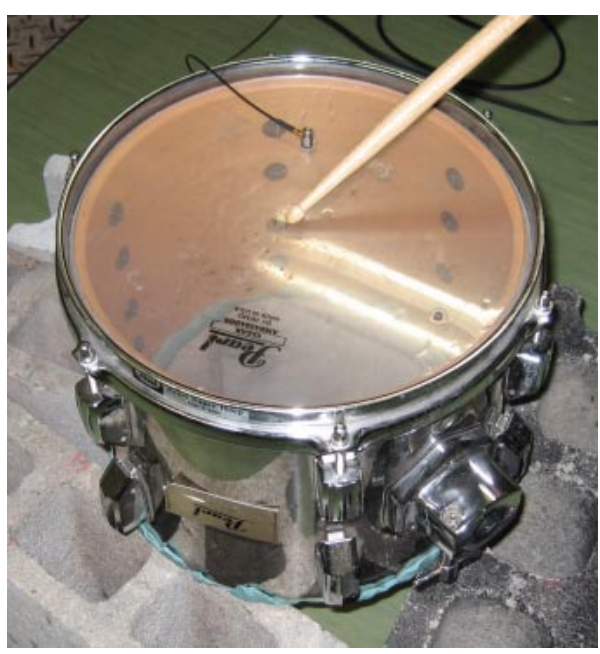

Fig. 8 Typical drum tom tom with six tension rods.

\subsection{Conditions of the Analysis}

The membrane was fastened along its boundary circle. The radius, $a$, of the membrane was $0.1778 \mathrm{~m}$ and the uniform areal density, $\rho_{\mathrm{M}}$, was set as $0.3 \mathrm{~kg} / \mathrm{m}^{2}$. On the assumption that several tension rods are left to be loosened, we developed a mathematical function given by Eq. (16) to demonstrate the variation in membrane tension $T(\tilde{r}, \tilde{\theta})$,

$$
T(\tilde{r}, \tilde{\theta})=d_{0} T_{0}+\left\{d(\tilde{r})-d_{0}\right\} b(\tilde{\theta}) T_{0},
$$

with

$$
\begin{aligned}
& d(\tilde{r})=d_{0}\left(\frac{d_{1}}{d_{0}}\right)^{\tilde{r}}, \\
& b(\tilde{\theta})=\sum_{n=0}^{N} B_{n} \exp \left\{-\frac{(\tilde{\theta}-2 \pi n / N)^{2}}{2 q^{2}}\right\} .
\end{aligned}
$$

In order to construct the Fourier-Chebyshev differentiation matrix given by Eq. (10), the derivatives of $T(\tilde{r}, \tilde{\theta})$ with respect to $\tilde{r}$ and $\tilde{\theta}$ are required, and these are expressed as

$$
\begin{aligned}
& \frac{\partial T(\tilde{r}, \tilde{\theta})}{\partial \tilde{r}}=b(\tilde{\theta}) T_{0} \frac{\partial d(\tilde{r})}{\partial \tilde{r}}, \\
& \frac{\partial T(\tilde{r}, \tilde{\theta})}{\partial \tilde{\theta}}=-d_{0} T_{0} \frac{\partial b(\tilde{\theta})}{\partial \tilde{\theta}},
\end{aligned}
$$

with

$$
\begin{aligned}
& \frac{\partial d(\tilde{r})}{\partial \tilde{r}}=d_{0}\left(\frac{d_{1}}{d_{0}}\right)^{\tilde{r}} \ln \left(\frac{d_{1}}{d_{0}}\right), \\
& \frac{\partial b(\tilde{\theta})}{\partial \tilde{\theta}}=-\sum_{n=0}^{N} B_{n} \frac{(\tilde{\theta}-2 \pi n / N)}{q^{2}} \exp \left\{-\frac{(\tilde{\theta}-2 \pi n / N)^{2}}{2 q^{2}}\right\} .
\end{aligned}
$$

When $T_{0}=2,700 \mathrm{~N} / \mathrm{m}, d_{0}=1, d_{1}=1 / 3, q=\pi / 16, N=$ 6 (the number of tension rods), $B_{0}=B_{6}=1, B_{2}=B_{3}=$ $B_{4}=1 / 2$, and $B_{1}=B_{5}=0$, this function produces the variations in tension shown in Fig. 9. For these parameters, we assumed a situation where one tension rod at $\theta=0$ was loosened, and thus $B_{0}$ and $B_{6}$ were set to 1 ; this is considered to be the main perturbation of the tension. Since

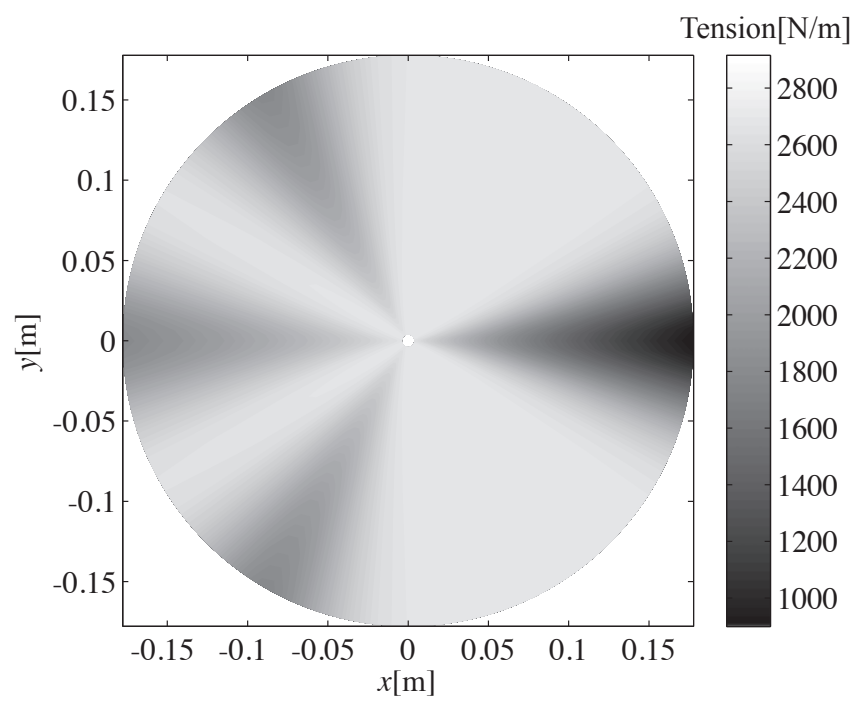

Fig. 9 Variation in tension of the membrane.

the net applied force at the perimeter of the membrane must equal zero as a necessary condition for equilibrium, the tension in other region is to be lowered. As an example of such a condition, $B_{2}, B_{3}$, and $B_{4}$ were set to $1 / 2$. We consider that although Eq. (16) is artificial, it could properly demonstrate the nonuniformity of the tension that stems from the assumed situation. However, in a strict sense, an isotropic condition would not produce this type of tension distribution. We expect that our preliminary computational model could grasp, in part, the dynamics concerned. We used this function for the tension $T(\tilde{r}, \tilde{\theta})$ to construct the Fourier-Chebyshev differentiation matrix given by Eq. (10). For mesh generation of the spectral method, $N_{r}=25$ and $N_{\theta}=30$ were used.

\subsection{Results}

In Table 2 the eigenfrequencies of the membrane with a uniform tension are compared with those of the membrane with a nonuniform tension calculated by the proposed numerical method. The uniform tension, $T$, of the membrane was set to $2,700 \mathrm{~N} / \mathrm{m}$. The theoretical eigenfrequencies in the uniform case are also presented. The theoretical and numerical eigenfrequencies in the uniform case are those presented in Table 1 without normalization. It is demonstrated that the degeneracy of even and odd modes of the nonuniform membrane is lifted, and thus each of these modes splits into two frequencies. This result is consistent with the experimental observations determined in a previous paper [6].

Figures 10(a) and 10(b) show the $(1,1)$ mode shapes calculated using the proposed numerical method. The even mode has a nodal line perpendicular to the main direction of perturbation (direction of $\theta=0$ ) in the applied tension that is an even function. Its eigenfrequency is lowered 
Table 2 Eigenfrequencies $[\mathrm{Hz}]$ of the membranes with uniform and nonuniform tension.

\begin{tabular}{llll}
\hline Modal Index & $\begin{array}{l}\text { Uniform } \\
\text { (Theoretical } \\
\text { values) }\end{array}$ & $\begin{array}{l}\text { Uniform } \\
\text { (Spectral } \\
\text { method) }\end{array}$ & $\begin{array}{l}\text { Non- } \\
\text { uniform } \\
\text { (Spectral } \\
\text { method) }\end{array}$ \\
\hline$(0,1)$ & 204.218 & 204.218 & 192.661 \\
$(1,1)_{\text {even }}$ & 325.388 & 325.388 & 305.053 \\
$(1,1)_{\text {odd }}$ & 325.388 & 325.388 & 311.623 \\
$(2,1)_{\text {even }}$ & 436.117 & 436.117 & 407.006 \\
$(2,1)_{\text {odd }}$ & 436.117 & 436.117 & 412.125 \\
$(0,2)$ & 468.765 & 468.765 & 445.845 \\
$(3,1)_{\text {even }}$ & 541.803 & 541.803 & 506.423 \\
$(3,1)_{\text {odd }}$ & 541.803 & 541.803 & 508.126 \\
$(1,2)_{\text {even }}$ & 595.763 & 595.763 & 554.320 \\
$(1,2)_{\text {odd }}$ & 595.763 & 595.763 & 572.295 \\
$(4,1)_{\text {even }}$ & 644.402 & 644.402 & 600.516 \\
$(4,1)_{\text {odd }}$ & 644.402 & 644.402 & 606.499 \\
$(2,2)_{\text {even }}$ & 714.792 & 714.792 & 656.302 \\
$(2,2)_{\text {odd }}$ & 714.792 & 714.792 & 677.676 \\
$(0,3)$ & 734.874 & 734.874 & 695.138 \\
\hline
\end{tabular}
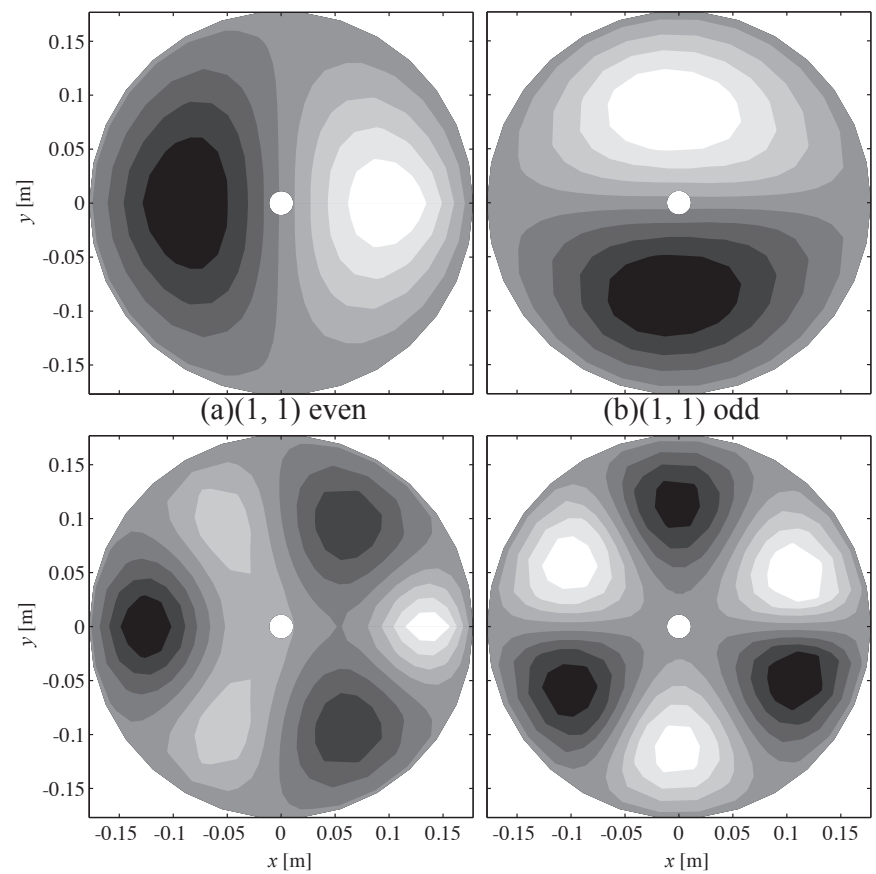

(c) $(3,1)$ even

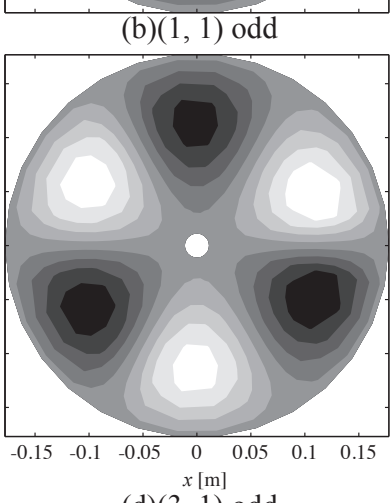

$(\mathrm{d})(3,1)$ odd

Fig. 10 Mode $(1,1)$ and $(3,1)$ shapes of the membrane with nonuniform tension. because waves traveling across the nodal line have an antinode in the part with lower tension. On the other hand, the odd mode, which has a nodal line that aligns with the main perturbation, does not lower much. It is possible to see that the nodal line of the lower frequency mode is slightly curved around the region where the tension was most lowered $(\theta=0)$. These results are also consistent with experimental observations described in the previous paper [6]. Figures 10(c) and 10(d) show the $(3,1)$ mode shapes calculated using the proposed method. In this case, the even mode shape can be seen as clearly deformed and the nodal lines are curved around the region where the tension was most lowered.

\section{SUMMARY}

In this work, the spectral method given by Sathej and Adhikari for membrane vibration analysis is extended to include nonuniformity of tension as well as that of density. The proposed numerical method uses a spectral method, and is able to efficiently analyze the membrane vibration of nonuniform drum heads. Numerical results are also consistent with those determined in previous experimental observations. Using the method, the authors aim to design a more attractive membranophone and to develop a theoretical process for use in tuning a drum. We could also find application in numerical sound synthesis based on more realistic numerical models of membranophones.

\section{REFERENCES}

[1] N. H. Fletcher and T. D. Rossing, The Physics of Musical Instruments (Springer-Verlag, New York, 1998), Chap. 18.5.

[2] N. H. Fletcher and T. D. Rossing, The Physics of Musical Instruments (Springer-Verlag, New York, 1998), Chap. 18.6.

[3] B. S. Ramakrishna and M. M. Sondhi, "Vibrations of Indian musical drums regarded as composite membranes," J. Acoust. Soc. Am., 26, 523-529 (1954).

[4] S. Ando and K. Yamaguchi, "Consideration on vibrations of Japanese traditional drums 'Tsuzumi'," J. Acoust. Soc. Jpn. (J), 41, 239-247 (1985) (in Japanese).

[5] G. Sathej and R. Adhikari, "The eigenspectra of Indian musical drums," J. Acoust. Soc. Am., 125, 831-838 (2009).

[6] R. Worland, "Normal modes of a musical drumhead under nonuniform tension," J. Acoust. Soc. Am., 127, 525-533 (2010).

[7] P. M. Morse, Vibration and Sound (McGraw-Hill, New York, 1948), Sect. 11.

[8] L. N. Trefethen, Spectral Methods in MATLAB (SIAM, Philadelphia, 2000). 